\title{
Interstitial Lung Disease Evaluation: Detecting Connective Tissue Disease
}

\author{
Aryeh Fischer $^{\mathrm{a}} \quad$ Joyce S. Lee ${ }^{\mathrm{a}}$ Vincent Cottin ${ }^{\mathrm{b}}$ \\ ${ }^{a}$ Department of Medicine, University of Colorado School of Medicine, Aurora, Colo., USA; b Service de \\ Pneumologie, Reference Center for Rare Pulmonary Diseases, Louis Pradel Hospital, Claude Bernard University \\ Lyon 1, Lyon, France
}

\section{Key Words}

Connective tissue disease $\cdot$ Interstitial lung disease . Interstitial pneumonia - Collagen vascular disease . Pulmonary fibrosis

\begin{abstract}
Interstitial lung disease (ILD) is a common manifestation of connective tissue disease (CTD) and is often associated with significant morbidity and mortality. The evaluation of ILD in patients with CTD is complex because of the heterogeneity of the CTD spectrum, the various patterns and degrees of severity of ILD encountered, and because ILD can be identified at any point in time in these patients. A thorough - and optimally multidisciplinary - evaluation is needed when CTD patients develop ILD or when evaluating ILD patients for the presence of occult CTD. Determining whether ILD is associated with a preexisting CTD requires the exclusion of alternative etiologies, and thorough assessments of the clinical features of both the CTD and ILD. The detection of occult CTD in patients with presumed idiopathic interstitial pneumonia requires careful integration of clinical, serologic, and thoracic imaging and histopathologic features.
\end{abstract}

(c) 2015 S. Karger AG, Basel

Previous articles in this series: 1. Bouros D, Papiris S, Cottin V: Lung involvement in rheumatic disease: introduction. Respiration 2015;90:1. 2. Papiris SA, Manali ED, Kolilekas L, Kagouridis K, Maniati M, Borie R, Pradere $P$, Crestani $B$, Bouros D: Investigation of lung involvement in connective tissue disorders. Respiration 2015;90:2-24.
(C) 2015 S. Karger AG, Basel

0025-7931/15/0903-0177\$39.50/0

\section{Introduction}

Interstitial lung disease (ILD) encompasses a spectrum of diffuse parenchymal lung disorders classified together because of shared clinical, radiological, and histopathological features [1-3]. Although most often a cause is not identified, and the ILD is considered to be 'idiopathic' in nature; frequently, ILD arises within the context of a specific exposure or is associated with an underlying connective tissue disease (CTD). The CTDs are a group of systemic autoimmune disorders (also referred to as 'collagen vascular diseases') characterized by immune-mediated organ dysfunction, and significant clinical heterogeneity exists within this spectrum (table 1). Typically, CTD manifests with autoimmune-mediated organ damage and the lung is a frequent target. Although all patients with CTD are at risk of developing ILD, certain CTDs are more frequently associated with ILD [e.g., systemic sclerosis (SSc) and polymyositis/dermatomyositis (PM/ $\mathrm{DM})]$, and there can be differing patterns of clinical, imaging, and histologic presentation [4].

Precise prevalence rates of ILD among the various CTDs are not known and are influenced by the methods of detection [5]. For example, studies that base the definition of the presence of ILD on a plain chest roentgenogram will cite a lower prevalence rate compared with studies that define the presence of ILD by thoracic highresolution computed tomography (HRCT). Although ILD has been a well-known manifestation of CTD, perhaps because of the technological advances in diagnostic

\section{KARGER 125}

E-Mail karger@karger.com www.karger.com/res
Aryeh Fischer, MD

Department of Medicine, University of Colorado School of Medicine 1775 Aurora Court, PO Box 6511, Mail Stop B-115 Aurora, CO 80045 (USA)

E-Mail aryeh.fischer@ucdenver.edu 
Table 1. CTDs (or 'collagen vascular diseases')

Systemic lupus erythematosus

Systemic sclerosis

Sjögren's syndrome

Rheumatoid arthritis

Polymyositis/dermatomyositis

Mixed connective tissue disease

Undifferentiated connective tissue disease

imaging - with the advent of HRCT in particular, the prevalence of ILD in CTD may be higher than previously considered [5], but only a fraction of these individuals have symptoms (such as exertional dyspnea) related to the ILD that would be considered as clinically significant disease [6].

ILD may be recognized at any point in the natural history of CTD [7]. Most often ILD is identified within the context of an established CTD, but there is a growing appreciation that ILD may be the first clinically apparent manifestation of an occult CTD (i.e., forme fruste presentation) [8-10]. Furthermore, some individuals may have interstitial pneumonia with autoimmune features (IPAF), as defined by the presence of ILD and features suggestive of - but not diagnostic for - an underlying CTD [11].

Because of the potential impact on both treatment and prognosis, determining whether a patient's ILD is associated with CTD is important - and yet the evaluation for CTD can be particularly challenging. In this focused review, we discuss our approach to the evaluation of ILD in those with established CTD and to the detection of occult CTD (or IPAF) in those with presumed idiopathic interstitial pneumonia (IIP).

\section{Relevance of the Diagnosis}

Determining whether a patient has a diagnosis of CTDassociated ILD (CTD-ILD) - rather than an IIP - may impact treatment decisions, guide surveillance for other concomitant clinical features, and ultimately influence prognosis. From the aspects of therapeutics, the importance of this distinction is likely most significant when the underlying ILD pattern is usual interstitial pneumonia (UIP). A clinical diagnosis of idiopathic pulmonary fibrosis (IPF) is restricted to those with a pattern of UIP in whom no identifiable etiology has been identified $[1,2]$. As such, concluding that a patient has IPF requires a comprehensive and often multidisciplinary evaluation to ensure that all identifiable etiologies (e.g., CTD) have been reliably excluded. With the recent approval of two novel anti-fibrotic drugs proven to only be effective in IPF [12, 13 ], and as such, restricted exclusively to this scenario this distinction perhaps has never been more important. Indeed, there is a pressing need to conduct clinical trials to evaluate whether these novel anti-fibrotic drugs might be useful in patients with CTD-ILD. Further, in contrast to IPF in which immunosuppression has been proven either ineffective or potentially harmful [14-16], there are data to suggest that systemic immunosuppressive medications may favorably impact CTD-ILD in general (and potentially even those with CTD-associated UIP) $[17,18]$.

For those with non-UIP patterns, the importance of distinguishing idiopathic from identifiable etiology is important as well. For example, if the ILD is determined to be drug-induced, the offending drug is discontinued. If due to an environmental allergen, the offending antigen is removed by an environmental inspection and remediation. And if the underlying cause of the ILD is determined to be an associated CTD, there will likely be a need for long-term systemic immunomodulatory therapy.

Identifying that an underlying CTD is present may help in the clinical care of patients as this knowledge may provide a clinical explanation for otherwise unexplained extrathoracic manifestations (e.g., Raynaud's phenomenon or a specific dermatitis) or lend to more vigilant assessment for other common disease manifestations (e.g., esophageal reflux and hypomotility). In addition, identifying CTD may lead to implementation of recommended screening strategies for other severe disease features (e.g., pulmonary arterial hypertension screening in SSc or malignancy surveillance in $\mathrm{PM} / \mathrm{DM}$ ) that could lead to an earlier diagnosis and improved outcome as a result.

Finally, distinguishing CTD-ILD from IIP can help with prognostication, as those with CTD-ILD tend to have a more favorable prognosis compared with IIP [19], with the notable exception of patients with rheumatoid arthritis (RA) and a UIP pattern at imaging and/or biopsy that tend to have an outcome as severe as that of IPF [20,21].

\section{Evaluation: ILD in Established CTD}

In those that develop ILD within the context of an established, preexisting CTD, as with any patient that presents with interstitial infiltrates, a comprehensive evaluation is needed to explore all potential etiologies (e.g., infection, medication toxicity, environmental exposures, smoking-related lung disease, malignancy, familial disease, etc.). The determination that the ILD is associated 
with the established CTD is through a process of elimination and requires a comprehensive evaluation. When considering the evaluation of ILD in patients with established CTD, we often consider the following steps.

\section{Confirm the CTD}

It is important to ensure that the 'established' CTD is an accurate diagnosis. For example, does the patient really have RA? In those with symmetric synovitis, rheumatoid nodules, rheumatoid factor (RF) and anti-CCP positivity, and X-ray evidence of characteristic juxta-articular erosions, the diagnosis will be more straightforward. But what about the patient with longstanding arthritis that is $\mathrm{RF}$ and anti-CCP negative and lacks X-ray evidence of joint erosions? Does that patient really have a diagnosis of seronegative, nonerosive RA? Or what about the patient with keratoconjuctivitis sicca (KCS) and labeled for years with a diagnosis of primary Sjögren's syndrome $(\mathrm{SjS})$ ? Sometimes the scenario is straightforward, such as when there is KCS accompanied by objective findings of autoantibody positivity (ANA, SSA, SSB, RF), a polyclonal gammopathy, and lymphocytic sialadenitis on labial salivary gland biopsy. But what about the patient with symptoms of KCS and a positive ANA? How certain should we be that this latter patient has 'established' SjS? In other words, sometimes the precise rheumatologic diagnosis may be uncertain and the development of ILD may impact its classification. Or the finding of ILD may lead to reevaluation of whether the patient really has an 'established' CTD. Take for instance the patient with Raynaud's phenomenon, rash, a low-titer ANA and hightiter SSA autoantibodies that has been considered to have an established diagnosis of systemic lupus erythematosus. If the patient then develops nonspecific interstitial pneumonia (NSIP) with overlapping organizing pneumonia $(\mathrm{OP})$, one might consider the manifestations to be associated with an anti-synthetase antibody and the anti-synthetase syndrome, rather than what was initially suspected - in the absence of chronic ILD - to be more likely a case of systemic lupus erythematosus.

\section{Determine whether the ILD Pattern Is Consistent with CTD-ILD}

The knowledge that a patient has a CTD should lead to heightened awareness for the possibility of coexistent ILD and presents an opportunity for earlier diagnosis - perhaps even as subclinical disease [6]. Thoracic HRCT imaging plays an integral role in the evaluation of ILD by providing detailed information on the pattern, distribution and extent of the ILD, assessment of disease severity, and the presence of extraparenchymal abnormalities [22-24]. Individuals with CTD are also more likely to have an HRCT pattern suggestive of NSIP when compared to those without CTD. Compared with IIP, patients with CTD-ILD are more likely to have ground glass abnormalities, pleural effusions, pericardial effusions, pericardial thickening, and esophageal dilatation [22]. And although as a cohort, those with CTD-ILD are less likely to have honeycombing, it is important to remember that UIP is the most common ILD pattern in RA and is identified across the spectrum of CTD. Overlapping patterns consistent with UIP and NSIP or NSIP and OP are not unusual and can be considered almost routine in disorders such as $\mathrm{PM} / \mathrm{DM}$. More unusual patterns such as lymphocytic interstitial pneumonia (LIP) with cystic lung disease (e.g., especially with $\mathrm{SjS}$ ) and primary airway disease (e.g., bronchiolitis) may also occur in specific settings.

\section{Consider Alternative Etiologies}

A comprehensive evaluation is needed in all patients that develop ILD - and having an established CTD does not mitigate this requirement. As such, it is important to consider and evaluate for infection, hypersensitivity pneumonitis, drug toxicity, malignancy, smoking-related lung disease, etc., before concluding that the ILD is CTDrelated. In particular, respiratory infection and drug-induced lung disease are almost always in the differential diagnosis and require thorough assessment.

\section{Perform Bronchoalveolar Lavage or Transbronchial \\ Biopsy when Clinically Appropriate}

The routine use of bronchoalveolar lavage (BAL) to solely predict the likelihood of disease progression in CTD-ILD is no longer recommended. However, because the CTD patient is often immunocompromised, BAL can be useful to exclude infection. Furthermore, BAL can be useful when diffuse alveolar hemorrhage is a concern. Transbronchial biopsy is of limited value in the evaluation of ILD in CTD, but may be diagnostic in more airway-centric complications such as bronchiolitis, hypersensitivity pneumonitis, sarcoidosis, or malignancy.

\section{Consider Surgical Lung Biopsy}

There are limited data on which to base definite comments about the impact of histopathology on prognosis in those with CTD-ILD. Further, the existing data are largely from specialty referral centers, consist of small cohorts of patients, and as such preclude the ability to draw firm conclusions. However, because the available data have yet to convincingly show that determining a specific histo- 
pathologic pattern of lung injury directly impacts prognosis in CTD-ILD $[19,25]$, the role of surgical lung biopsy in patients with preexisting CTD remains controversial. In contrast to IIP, the distinction between the specific ILD subtypes (e.g., UIP vs. NSIP) may not be as prognostically significant in patients with CTD. One exception appears to be with respect to RA and a UIP pattern. Recent data from several cohorts of RA-ILD suggest that survival in RA-UIP may be similar to that of IPF and worse than that seen in CTD-NSIP or other non-RA-CTD-UIP [20]. Further, although the correlation between HRCT and the histologic pattern is not as strong as in IIP, there is a growing appreciation that a UIP pattern on HRCT in the context of CTD is predictive of histologic UIP [26].

Another reason why CTD-ILD patients infrequently undergo surgical lung biopsy is that these patients tend to be treated with systemic immunosuppressive therapies targeting both progressive ILD and the extrathoracic inflammatory features - irrespective of the specific ILD pattern. In this context, because the biopsy findings may not impact treatment decisions, when the HRCT provides a strongly suggestive pattern consistent with what would be expected for CTD, clinicians often elect not to proceed with a surgical lung biopsy.

In our opinion, a surgical lung biopsy may be appropriate in patients with preexisting CTD when there are significant concerns for an alternative etiology (e.g., hypersensitivity pneumonitis or smoking-related lung disease), when the HRCT is 'atypical' for underlying CTD (e.g., upper lobe predominant disease), when it suggests malignancy or infection (e.g., progressive nodules, cavitation, consolidation, pleural thickening, or effusion), or when the diagnosis of CTD is still unclear. Ultimately, the decision of whether to perform a surgical lung biopsy is individualized, with due consideration for its associated risks and whether its findings will impact management and prognosis [27].

\section{The ILD Evaluation: Identifying Forme Fruste CTD}

Identifying occult CTD among those with a presumed 'idiopathic' interstitial pneumonia is common. Although the precise frequency by which occult CTD is detected among those presenting with ILD is not known, a recent study from a multidisciplinary ILD program reported that of 114 consecutive ILD patients evaluated, 17 (15\%) were confirmed to have a new CTD diagnosis [28].

There is no standardized approach to the evaluation of CTD in ILD, and current guidelines recommend performing a thorough history and physical examination
Table 2. Suggested categories of ILD patients that require further rheumatologic evaluation [30]

1. Women, particularly those younger than 50 years

2. Any patient with extrathoracic manifestations highly suggestive of CTD

i.e., Raynaud's phenomenon, esophageal hypomotility, inflammatory arthritis of the metacarpal-phalangeal joints or wrists, digital edema, or symptomatic KCS

3. All cases of NSIP, LIP, or any ILD pattern with secondary histopathology features that might suggest CTD i.e., extensive pleuritis, dense perivascular collagen, lymphoid aggregates with germinal center formation, prominent plasmacytic infiltration

4. Patients with a positive ANA or RF in high titer (generally considered to be ANA >1:320 or RF $>60 \mathrm{IU} / \mathrm{ml}$ ), a nucleolarstaining ANA at any titer, or any positive autoantibody specific as to a particular CTD

i.e., anti-CCP, anti-Scl-70, anti-Ro, anti-La, anti-dsDNA, anti-Smith, anti-RNP, anti-tRNA synthetase

and testing for a panel of circulating autoantibodies $[1,2]$. Many centers have also found that a multidisciplinary evaluation that includes rheumatologic consultation is useful $[29,30]$. However, because it is both unrealistic and impractical to have rheumatologic specialty evaluation for all cases of IIP, it is left up to the individual provider to decide when to obtain rheumatologic consultation. In general, it may be reasonable to engage rheumatologists in scenarios in which there is a significant index of suspicion for underlying CTD, and to this aim, certain guidelines have been suggested [30] (table 2).

Ultimately, confirming a diagnosis of CTD requires the integration of clinical features, serologies, and thoracic morphologic features identified by imaging and/or histopathology. Ideally, the evaluation is comprehensive and multidisciplinary in nature.

\section{Clinical Features}

Demographic features can help distinguish the patient with an underlying CTD. In comparison to IPF, patients with CTD-ILD are more likely to be younger and female. A comprehensive history, review of systems, and thorough physical examination with careful attention to systemic symptoms and signs are essential components of the evaluation. Certain specific clinical features lend more support for underlying CTD than others. Of the CTD symptoms encountered in patients with IIP, perhaps none is as important as Raynaud's phenomenon. The presence of Raynaud's phenomenon is associated with a pattern of 
NSIP and when identified in a patient with ILD should raise strong suspicions for underlying CTD in general, and SSc (with or without overt skin thickening) or myositis spectrum in particular. Indeed, Raynaud's phenomenon is encountered in nearly all patients with SSc and is also a particularly common finding in patients with PM/ DM among other CTDs. Performing nailfold capillary microscopy can be useful when assessing a patient with Raynaud's phenomenon. The presence of dilated or tortuous capillary loops or significant areas lacking capillary loops (i.e., capillary dropout) may be particularly helpful in the assessment of forme fruste SSc or PM/DM.

Symptoms of symmetric joint swelling or stiffness, or identifying synovitis on physical examination are also very useful. Because inflammatory arthritis may be encountered in all of the CTDs, other clinical clues or autoantibody profiles are often helpful to clarify which specific CTD is present. In contrast to the specific features described, symptoms such as gastroesophageal reflux, pain, fatigue, sicca syndrome, alopecia, or weight loss are not nearly as helpful because they are so commonly reported and are far less specific for CTD.

The cutaneous manifestations of SSc, the myositis-SSc overlap associated with PM-Scl antibody, and the socalled anti-synthetase syndrome (also associated with anti-PM-Scl antibodies) are worthy of a special mention because these disorders are so commonly associated with ILD and their extrathoracic features are both specific and often subtle. The 'mechanic hands' of anti-synthetase syndrome or those of patients with the PM-Scl antibody can be as subtle as showing only mild distal digital fissuring, and palmar telangiectasia may be limited to the finding of only few scattered dilated capillaries. Nonetheless, when such findings are present in a patient with an IIP, they are highly suggestive of underlying CTD $[9,31,32]$.

\section{Circulating Autoantibodies}

Autoantibody assessment can be an important part of the evaluation of patients with IIP but also requires clinical interpretation and sometimes leads to more confusion than clarity. For patients with ILD in whom there is clinical suspicion of an underlying CTD, a broad panel of autoantibodies is recommended to ensure that the full spectrum of CTD is encompassed serologically (table 3). The preferred method for the ANA assay is by indirect immunofluorescence which allows for evaluating ANA titer and staining pattern [33]. The ELISA assay for ANA testing is less reliable, has been shown to be falsely negative in subsets of patients with SSc, does not allow for staining pattern reporting, and does not provide a titer
Table 3. Useful antibodies for CTD-ILD assessment [30]

\begin{tabular}{ll}
\hline Autoantibody & Commonly associated CTD \\
\hline High-titer ANA $(\geq 1: 320$ titer) & Many \\
High-titer RF $(\geq 60 \mathrm{IU} / \mathrm{ml})$ & RA, SjS, SLE \\
Anti-CCP & RA \\
Anti-centromere & SSc \\
Anti-nucleolar ANA & SSc \\
Anti-Ro (SSA) & Many \\
Anti-La (SSB) & SLE, SjS \\
Anti-Smith & SLE \\
Anti-ribonucleoprotein & SLE, MCTD \\
Anti-dsDNA & SLE \\
Anti-topoisomerase (Scl-70) & SSc \\
Anti-tRNA synthetase antibodies & PM/DM \\
& (anti-synthetase syndrome) \\
Anti-PM-Scl & SSc/myositis overlap \\
Anti-Th/To & SSc \\
Anti-U3 ribonucleoprotein & SSc \\
Anti-MDA-5 (CADM) & Clinical amyopathic \\
& dermatomyositis \\
\hline
\end{tabular}

SLE = Systemic lupus erythematosus; $\mathrm{MCTD}=$ mixed connec tive tissue disease.

[34]. The pattern of immunofluorescence when the ANA is positive can be helpful, as the nucleolar-staining ANA pattern in patients with ILD may suggest an SSc spectrum of disease $[31,35]$, and the serendipitous observation of cytoplasmic fluorescence may suggest the presence of anti-synthetase antibodies [36, 37].

Among those with NSIP and/or OP, one should consider analyzing a myositis antibody panel that includes the family of tRNA synthetase antibodies, as the myositis spectrum is one of the more common forms of CTD-ILD. Further, it is important to note that many of these patients are amyopathic (i.e., lack chemical or clinical evidence of myositis) in nature and are often ANA negative. As such, a negative ANA test does not exclude the anti-synthetase syndrome. Further, although individuals with anti-synthetase syndrome are often ANA negative, they may have a positive anti-SSA antibody along with a confirmatory anti-tRNA synthetase antibody (e.g., Jo-1, PL-7, PL-12, etc.) $[36,37]$. It may be useful to test for rarer autoantibodies (e.g., anti-Mi2, MDA5, NXP2, and others) as part of a second-tier investigation in cases suggestive of PM/ $\mathrm{DM}$ when the anti-synthetase antibodies are negative.

Importantly, we highlight that the ANA and RF are relatively poor screening tests: they have low specificity - particularly when present at low titer - and can be seen in healthy individuals $[38,39]$. In addition, given that a negative ANA and RF may dissuade some clinicians 
Table 4. Classification criteria for IPAF [11]

1. Presence of an interstitial pneumonia (by HRCT or surgical lung biopsy) and

2. Exclusion of alternative etiologies and

3. Does not meet criteria of a defined CTD and

4. At least one feature from at least two of these domains:
A. Clinical domain
B. Serologic domain
C. Morphologic domain

A. Clinical domain

1. Distal digital fissuring (i.e., 'mechanic hands')

2. Distal digital tip ulceration

3. Inflammatory arthritis or polyarticular morning joint stiffness $>60 \mathrm{~min}$

4. Palmar telangiectasia

5. Raynaud's phenomenon

6. Unexplained digital edema

7. Unexplained fixed rash on the digital extensor surfaces (Gottron's sign)

B. Serologic domain

1. ANA $\geq 1: 320$ titer, diffuse, speckled, homogeneous patterns or

a. ANA nucleolar pattern (any titer) or

b. ANA centromere pattern (any titer)

2. $\mathrm{RF} \geq 2 \times \mathrm{ULN}$

3. Anti-CCP

4. Anti-dsDNA

5. Anti-Ro (SSA)

6. Anti-La (SSB)

7. Anti-ribonucleoprotein

8. Anti-Smith

9. Anti-topoisomerase (Scl-70)

10. Anti-tRNA synthetase (e.g., Jo-1, PL-7, PL-12; others are: EJ, OJ, KS, Zo, tRS)

11. Anti-PM-Scl

12. Anti-MDA-5

from pursuing further evaluation, cases of occult CTD that may be ANA and RF negative are missed.

\section{Thoracic Morphologic Features}

As discussed earlier, thoracic HRCT imaging plays a central role in the evaluation of ILD. The patterns of NSIP, OP, NSIP with OP, and LIP are commonly encountered in CTD-ILD, and as such, their presence should raise the suspicion for an underlying autoimmune process. A radiologic pattern of UIP is seen in CTD as well (particularly in RA), but may be less specific for CTD compared to the aforementioned patterns.

The evaluation of lung tissue obtained by surgical lung biopsy may also provide clues about whether an underlying CTD is present. Histopathologic features considered
C. Morphologic domain

1. Suggestive radiology patterns by HRCT (see text for descriptions)
a. NSIP
b. OP
c. NSIP with OP overlap
d. LIP

2. Histopathology patterns or features by surgical lung biopsy
a. NSIP
b. OP
c. NSIP with OP overlap
d. LIP
e. Interstitial lymphoid aggregates with germinal centers
f. Diffuse lymphoplasmacytic infiltration (with or without lymphoid follicles)
3. Multicompartment involvement (in addition to IP)
a. Unexplained pleural effusion or thickening
b. Unexplained pericardial effusion or thickening
c. Unexplained intrinsic airway disease ${ }^{1}$ (by PFTs, imaging, or pathology)
d. Unexplained pulmonary vasculopathy

IP = Interstitial pneumonia; PFTs = pulmonary function tests. ${ }^{1}$ Includes airflow obstruction, bronchiolitis or bronchiectasis.

to be highly associated with (though not specific for) CTD include the patterns of NSIP, OP, and LIP and the secondary features of interstitial lymphoid aggregates with germinal centers and diffuse lymphoplasmacytic infiltration with or without lymphoid follicles [40]. A histopathologic pattern of UIP is seen in CTD as well, but may be less specific for CTD compared to the aforementioned patterns. Importantly, when compared to IPF, those with CTD-UIP have fewer fibroblast foci and less honeycombing but have more germinal center formation and more evidence of inflammation $[41,42]$.

The presence of thoracic multicompartment involvement may provide additional clues that an occult CTD is present as this is another characteristic encountered among patients with CTD [11]. Unexplained intrinsic airway disease (e.g., bronchiolitis or bronchiectasis) is a common finding in CTD patients, especially those with RA and SjS and may be seen in the setting of CTD-ILD as well. Unexplained pleural or pericardial effusion or thickening should raise suspicions for occult CTD. Finally, unexplained pulmonary vasculopathy is associated with CTD, particularly SSc or mixed connective tissue disease. Though its presence is not diagnostic of CTD, as indeed, pulmonary hypertension is also frequently noted in IIP, 
when group 1 pulmonary arterial hypertension is present along with ILD, or when pulmonary hypertension is severe (mean pulmonary artery pressure $>35 \mathrm{~mm} \mathrm{Hg}$ by right heart catheter), it does lend additional support for the possibility of an occult CTD [11].

\section{Interstitial Pneumonia with Autoimmune Features}

Recent studies have demonstrated that some patients considered to have an IIP actually have certain, often subtle, clinical features that suggest an underlying autoimmune process and yet do not meet established diagnostic criteria for any of the characterizable CTDs [11, 43-47]. In some individuals, these features may occur in the absence of serologic abnormalities, while in others, a highly specific serum autoantibody may be present without typical systemic or extrathoracic findings. In some patients, the radiologic or histopathologic features may suggest an underlying CTD, but the absence of extrathoracic clinical or serologic findings precludes reliable classification as anything other than IIP. In other words, although the patient may have an 'autoimmune flavor', a diagnosis of CTD is not rendered due to a lack of specific characterizable features of a defined CTD - and the patient is considered to have an IIP by default. Differing but overlapping criteria and terms have been proposed to describe these patients, including 'undifferentiated CTD-associated ILD' [43, 46], 'lung-dominant CTD' [44], or 'autoimmune-featured ILD' [47]. Because of their different diagnostic criteria, each set would include many of, but not all, the same patients. The lack of consensus has been considered a major obstacle to the ability to conduct prospective studies needed to answer fundamental questions about these patients.

With an aim of addressing the current impasses, the European Respiratory Society and American Thoracic Society Task Force on Undifferentiated Forms of CTD-
ILD proposed the name interstitial pneumonia with autoimmune features' (IPAF) be used to identify individuals with ILD and features suggestive of a CTD that do not meet established classification criteria for a characterizable CTD [11] (table 4). The classification of IPAF requires the a priori exclusion of alternative etiologies for ILD - including any of the characterizable CTDs - along with the presence of at least one feature from at least two of three primary domains: clinical, serologic, and intrathoracic morphology (table 4). Future research investigations of a more uniform cohort are needed to determine the natural history and clinical implications of an IPAF classification.

\section{Conclusion}

ILD is a common manifestation of CTD and is associated with significant morbidity and mortality. The evaluation of ILD in patients with CTD is complex because of the heterogeneity of the CTDs, the varied types and degrees of severity of ILD encountered, and because ILD can be identified at any point in time in these patients. A thorough and optimally multidisciplinary - evaluation is needed when CTD patients develop ILD or when evaluating ILD patients for the presence of occult CTD. Determining whether ILD is associated with a preexisting CTD requires the exclusion of alternative etiologies, and thorough assessments of the clinical features of both the CTD and ILD. The detection of occult CTD in patients with presumed IIP requires careful attention to the demographic profile, historical clues, subtle physical examination findings, serologic profile, and radiologic and histopathologic features. Research investigations of patient cohorts with IPAF - a novel classification that encompasses individuals with ILD and features suggestive of a CTD - are needed to determine its clinical implications and natural history.

\section{References}

1 ATS/ERS: American Thoracic Society/European Respiratory Society International Multidisciplinary Consensus Classification of the Idiopathic Interstitial Pneumonias. This joint statement of the American Thoracic Society (ATS), and the European Respiratory Society (ERS) was adopted by the ATS board of directors, June 2001 and by the ERS Executive Committee, June 2001. Am J Respir Crit Care Med 2002;165:277-304.

-2 Raghu G, Collard HR, Egan JJ, Martinez FJ, Behr J, Brown KK, et al: An official ATS/ERS/
JRS/ALAT statement: idiopathic pulmonary fibrosis: evidence-based guidelines for diagnosis and management. Am J Respir Crit Care Med 2011;183:788-824.

- 3 Travis WD, Costabel U, Hansell DM, King TE Jr, Lynch DA, Nicholson AG, et al: An official American Thoracic Society/European Respiratory Society statement: update of the international multidisciplinary classification of the idiopathic interstitial pneumonias. Am J Respir Crit Care Med 2013;188: 733-748. 
-4 Fischer A, du Bois R: Interstitial lung disease in connective tissue disorders. Lancet 2012; 380:689-698.

5 Castelino FV, Varga J: Interstitial lung disease in connective tissue diseases: evolving concepts of pathogenesis and management. Arthritis Res Ther 2010;12:213.

6 Doyle TJ, Hunninghake GM, Rosas IO: Subclinical interstitial lung disease: why you should care. Am J Respir Crit Care Med 2012; 185:1147-1153.

7 Cottin V: Interstitial lung disease. Eur Respir Rev 2013;22:26-32.

$\checkmark 8$ Cottin V: Interstitial lung disease: are we missing formes frustes of connective tissue disease? Eur Respir J 2006;28:893-896.

$\$ 9$ Tzelepis GE, Toya SP, Moutsopoulos HM: Occult connective tissue diseases mimicking idiopathic interstitial pneumonias. Eur Respir J 2008;31:11-20.

10 Fischer A, Brown KK: Interstitial lung disease in undifferentiated forms of connective tissue disease. Arthritis Care Res (Hoboken) 2015; 67:4-11.

-11 Fischer A, Antoniou KM, Brown KK, Cadranel J, Corte TJ, du Bois RM, et al: An official European Respiratory Society/American Thoracic Society research statement: interstitial pneumonia with autoimmune features. Eur Respir J 2015, Epub ahead of print.

$\checkmark 12$ King TE Jr, Bradford WZ, Castro-Bernardini S, Fagan EA, Glaspole I, Glassberg MK, et al: A phase 3 trial of pirfenidone in patients with idiopathic pulmonary fibrosis. N Engl J Med 2014;370:2083-2092.

13 Richeldi L, du Bois RM, Raghu G, Azuma A, Brown KK, Costabel U, et al: Efficacy and safety of nintedanib in idiopathic pulmonary fibrosis. N Engl J Med 2014;370:2071-2082.

14 Raghu G, Anstrom KJ, King TE Jr, Lasky JA, Martinez FJ: Prednisone, azathioprine, and $\mathrm{N}$-acetylcysteine for pulmonary fibrosis. $\mathrm{N}$ Engl J Med 2012;366:1968-1977.

15 Raghu G, Brown KK, Costabel U, Cottin V, du Bois RM, Lasky JA, et al: Treatment of idiopathic pulmonary fibrosis with etanercept: an exploratory, placebo-controlled trial. Am J Respir Crit Care Med 2008;178:948-955.

16 Collard HR, Ryu JH, Douglas WW, Schwarz MI, Curran-Everett D, King TE Jr, et al: Combined corticosteroid and cyclophosphamide therapy does not alter survival in idiopathic pulmonary fibrosis. Chest 2004;125:2169-2174.

17 Fischer A, Krishnamoorthy M, Olson AL, Solomon JJ, Fernandez-Perez ER, Huie TJ, et al: Mycophenolate mofetil (MMF) in various interstitial lung diseases (abstract). Am J Respir Crit Care Med 2012;185:A3638.

-18 Tashkin DP, Elashoff R, Clements PJ, Goldin J, Roth MD, Furst DE, et al: Cyclophosphamide versus placebo in scleroderma lung disease. N Engl J Med 2006;354:2655-2666.

-19 Park JH, Kim DS, Park IN, Jang SJ, Kitaichi $\mathrm{M}$, Nicholson AG, et al: Prognosis of fibrotic interstitial pneumonia: idiopathic versus collagen vascular disease-related subtypes. Am J Respir Crit Care Med 2007;175:705-711.
20 Kim EJ, Collard HR, King TE Jr: Rheumatoid arthritis-associated interstitial lung disease: the relevance of histopathologic and radiographic pattern. Chest 2009;136:1397-1405.

21 Kim EJ, Elicker BM, Maldonado F, Webb WR, Ryu JH, Van Uden JH, et al: Usual interstitial pneumonia in rheumatoid arthritis-associated interstitial lung disease. Eur Respir J 2010;35:1322-1328.

22 Hwang JH, Misumi S, Sahin H, Brown KK, Newell JD, Lynch DA: Computed tomographic features of idiopathic fibrosing interstitial pneumonia: comparison with pulmonary fibrosis related to collagen vascular disease. J Comput Assist Tomogr 2009;33: 410-415.

23 Lynch DA: Quantitative CT of fibrotic interstitial lung disease. Chest 2007;131:643-644.

24 Lynch DA, Travis WD, Muller NL, Galvin JR, Hansell DM, Grenier PA, et al: Idiopathic interstitial pneumonias: CT features. Radiology 2005;236:10-21.

25 Kowal-Bielecka O, Kowal K, Highland KB, Silver RM: Bronchoalveolar lavage fluid in scleroderma interstitial lung disease: technical aspects and clinical correlations: review of the literature. Semin Arthritis Rheum 2012; 40:73-88.

26 Chung JH, Chawla A, Peljto AL, Cool CD, Groshong SD, Talbert JL, et al: CT scan findings of probable usual interstitial pneumonitis have a high predictive value for histologic usual interstitial pneumonitis. Chest 2015; 147:450-459.

27 Fischer A, Chartrand S: Assessment and management of connective tissue disease-associated interstitial lung disease. Sarcoidosis Vasc Diffuse Lung Dis 2015;32:2-21.

28 Mittoo S, Gelber AC, Christopher-Stine L, Horton MR, Lechtzin N, Danoff SK: Ascertainment of collagen vascular disease in patients presenting with interstitial lung disease. Respir Med 2009;103:1152-1158.

29 Fischer A, Richeldi L: Cross-disciplinary collaboration in connective tissue disease-related lung disease. Semin Respir Crit Care Med 2014;35:159-165.

-30 Fischer A, du Bois RM: A Practical Approach to Connective Tissue Disease-Associated Lung Disease, ed 2. New York, Springer, 2012.

- 31 Fischer A, Meehan RT, Feghali-Bostwick CA, West SG, Brown KK: Unique characteristics of systemic sclerosis sine scleroderma-associated interstitial lung disease. Chest 2006;130: 976-981.

32 Fischer A, Swigris JJ, du Bois RM, Lynch DA, Downey GP, Cosgrove GP, et al: Anti-synthetase syndrome in ANA and anti-Jo-1 negative patients presenting with idiopathic interstitial pneumonia. Respir Med 2009;103:17191724.

33 Solomon DH, Kavanaugh AJ, Schur PH; American College of Rheumatology Ad Hoc Committee on Immunologic Testing G: Evidence-based guidelines for the use of immunologic tests: antinuclear antibody testing. Arthritis Rheum 2002;47:434-444.
34 Shanmugam VK, Swistowski DR, Saddic N, Wang H, Steen VD: Comparison of indirect immunofluorescence and multiplex antinuclear antibody screening in systemic sclerosis. Clin Rheumatol 2011;30:1363-1368.

-35 Fischer A, Pfalzgraf FJ, Feghali-Bostwick CA, Wright TM, Curran-Everett D, West SG, et al: Anti-th/to-positivity in a cohort of patients with idiopathic pulmonary fibrosis. J Rheumatol 2006;33:1600-1605.

36 Lega JC, Cottin V, Fabien N, Thivolet-Bejui F, Cordier JF: Interstitial lung disease associated with anti-PM/Scl or anti-aminoacyl-tRNA synthetase autoantibodies: a similar condition? J Rheumatol 2010;37:1000-1009.

-37 Lega JC, Fabien N, Reynaud Q, Durieu I, Durupt $\mathrm{S}$, Dutertre $\mathrm{M}$, et al: The clinical phenotype associated with myositis-specific and associated autoantibodies: a meta-analysis revisiting the so-called antisynthetase syndrome. Autoimmun Rev 2014;13:883891.

38 Goodwin JS, Searles RP, Tung KS: Immunological responses of healthy elderly population. Clin Exp Immunol 1982;48:403-410.

39 Manoussakis MN, Tzioufas AG, Silis MP, Pange PJ, Goudevenos J, Moutsopoulos HM: High prevalence of anti-cardiolipin and other autoantibodies in a healthy elderly population. Clin Exp Immunol 1987;69:557-565.

40 Leslie KO, Trahan S, Gruden J: Pulmonary pathology of the rheumatic diseases. Semin Respir Crit Care Med 2007;28:369-378.

41 Flaherty KR, Colby TV, Travis WD, Toews GB, Mumford J, Murray S, et al: Fibroblastic foci in usual interstitial pneumonia: idiopathic versus collagen vascular disease. Am J Respir Crit Care Med 2003;167:1410-1415.

42 Song JW, Do KH, Kim MY, Jang SJ, Colby TV, Kim DS: Pathologic and radiologic differences between idiopathic and collagen vascular disease-related usual interstitial pneumonia. Chest 2009;136:23-30.

43 Corte TJ, Copley SJ, Desai SR, Zappala CJ, Hansell DM, Nicholson AG, et al: Significance of connective tissue disease features in idiopathic interstitial pneumonia. Eur Respir J 2012;39:661-668.

44 Fischer A, West SG, Swigris JJ, Brown KK, du Bois RM: Connective tissue disease-associated interstitial lung disease: a call for clarification. Chest 2010;138:251-256.

45 Fujita J, Ohtsuki Y, Yoshinouchi T, Yamadori I, Bandoh S, Tokuda M, et al: Idiopathic non-specific interstitial pneumonia: as an 'autoimmune interstitial pneumonia’. Respir Med 2005;99:234-240.

46 Kinder BW, Collard HR, Koth L, Daikh DI, Wolters PJ, Elicker B, et al: Idiopathic nonspecific interstitial pneumonia: lung manifestation of undifferentiated connective tissue disease? Am J Respir Crit Care Med 2007;176: 691-697.

47 Vij R, Noth I, Strek ME: Autoimmune-featured interstitial lung disease: a distinct entity. Chest 2011;140:1292-1299. 